\title{
Social Egg Freezing: Developing Countries Are Not Exempt
}

\author{
Gautam N. Allahbadia ${ }^{1}$
}

Published online: 13 November 2015

(C) Federation of Obstetric \& Gynecological Societies of India 2015

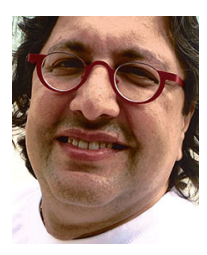

\begin{abstract}
About the Author
Gautam N Allahbadia, MD is the Editor-in-Chief of the Journal of Obstetrics \& Gynecology of India as well as the IVF Lite (Journal of Minimal Stimulation IVF). He is the Medical Director of Rotunda-The Center for Human Reproduction, the world-renowned infertility clinic at Bandra, and Rotunda-Blue Fertility Clinic and Keyhole Surgery Center, Mumbai, India. $\mathrm{He}$ is a noted world authority on ultrasound-guided embryo transfers and one of the pioneers in Third Party Reproduction in Southeast Asia. Dr. Allahbadia was responsible for India's first trans-ethnic surrogate pregnancy involving a Chinese couple's baby delivered by an unrelated Indian surrogate mother. He cherishes over 125 peer-reviewed publications, 134 book chapters and 22 textbooks, the latest being a comprehensive text, entitled "Minimal Stimulation IVF", and is on the Editorial Board of several International Journals. He just completed a two year tenure as Medical Director at the New Hope IVF Clinic, Sharjah, UAE, where he introduced IVF Lite to the country. Dr. Allahbadia has recently been elected as the Vice President of the World Association of Reproductive Medicine (WARM), headquartered in Rome, and "Mumbai's Top Doc" for 2012 by a peer nomination
\end{abstract} process. You can read more about his work at www.gautamallahbadia.com.

\begin{abstract}
Non-medical egg freezing has only been available for about the last 5 years, as new vitrification techniques have made the success rates for actual conception more reliable than the earlier method of slow freezing. The improved outcomes of new technologies of
\end{abstract}

Gautam N Allahbadia is the Editor-in-Chief of the Journal of Obstetrics \& Gynecology of India as well as the IVF Lite (Journal of Minimal Stimulation IVF) and Medical Director of Rotunda-The Center for Human Reproduction, Bandra, and Rotunda-Blue Fertility Clinic and Keyhole Surgery Center, Parel, Mumbai, India.

Gautam N. Allahbadia

ivfwaladoc@gmail.com

1 Rotunda-The Center for Human Reproduction, Mumbai, India vitrification and intra-cytoplasmic sperm injection (ICSI) have led to the marketing of egg freezing for non-medical reasons, whereby women are offered the possibility of preserving their eggs until such time as they wish to have a child. For many women today, it is not cancer but the simple passage of time that robs them of their chance of motherhood. Social, educational, emotional and financial pressures often lead them to delay trying to start a family until their late thirties, by which time the chance of success is very low. Women at age 40 face a $40 \%$ chance of miscarriage if they can get pregnant at all, and by the age of 45 , the risk of miscarriage is $75 \%$. Donor eggs are not an option for many because of supply constraints and ethical and cultural concerns. Freezing a woman's eggs at age 30 literally "freezes in time" her fertility potential and 
gives her the chance of a healthy pregnancy at a time of her choosing. Despite the initial reactions of disapproval, more and more fertility clinics are now offering oocyte cryopreservation to healthy women in order to extend their reproductive options. This procedure is now becoming popular even in developing economies, and egg freezing in major Indian Metros is now routine.

Keywords Social egg freezing - Oocytes .

Cryopreservation · Oocyte vitrification · Vitrification

\section{Introduction}

Cryopreservation itself has always played a central role in assisted reproductive technology. With the first cryopreservation of sperm in 1953 and of embryos thirty years later, these techniques have become routine. Dr Christopher Chen of Singapore reported the world's first pregnancy in 1986 using previously frozen oocytes [1]. Oocyte cryopreservation or egg freezing is aimed at three particular groups of women: those diagnosed with cancer who have not yet begun chemotherapy or radiotherapy; those undergoing treatment with assisted reproductive technologies who do not consider embryo freezing an option or where laws do not allow embryo freezing; and those who would like to preserve their future ability to have children, either because they do not yet have a partner, or for other personal or medical reasons.

Over 50,000 reproductive-age women are diagnosed with cancer each year in the USA [2]. The burden of cancer is increasing in economically developing countries as a result of population aging and growth as well as, increasingly, an adoption of cancer-associated lifestyle choices including smoking, physical inactivity and "westernized" diets. Chemotherapy and radiotherapy are toxic for oocytes, leaving few, if any, viable eggs. Egg freezing offers women with cancer the chance to preserve their eggs so that they can have children in the future.

Oocyte cryopreservation is an option for individuals undergoing IVF who object, either for religious or ethical reasons, to the practice of freezing embryos. Having the option to fertilize only as many eggs as will be utilized in the IVF process and then freeze any remaining unfertilized eggs can be a solution. In this way, there are no excess embryos created, and there need be no disposition of unused frozen embryos, a practice that can create complex choices for certain individuals.

Egg freezing can also be beneficial for women who, for the purpose of education, career or other reasons, desire to postpone childbearing. Freezing eggs at an early age may ensure a chance for a future pregnancy. Additionally, women with a family history of early menopause have an interest in fertility preservation. With egg freezing, they will have a frozen store of eggs, in the likelihood that their eggs are depleted at an early age.

Oocyte cryopreservation for age-related fertility loss is gaining interest considering the tendency to postpone motherhood in many societies. Little is currently known about the actual efficiency of this approach. Tsafrir et al. [3] aimed to explore ovarian response of presumably fertile women undergoing in vitro fertilization for this indication. A total of 105 women underwent 151 stimulation cycles at mean age $37.7 \pm 2.4$. None had known infertility. Mean daily starting FSH dose was $371 \pm 110$ (225-600). Mean number of mature oocytes cryopreserved at the first completed cycle was $9.7 \pm 7.5(0-43)$. However, $21 \%$ of started cycles were either canceled before egg retrieval or resulted in 0-3 mature oocytes retrieved. Therefore, women considering oocyte cryopreservation for prevention of agerelated fertility decline should be encouraged to perform this procedure at younger ages, preferably before 35 [3].

\section{Discussion}

Use of frozen thawed sperm has been in clinical use for over 50 years, and banking sperm has been routinely offered to men, usually before gonadotoxic treatments, but also in many cases, practiced as a "safety policy" before a vasectomy. Freezing methods for women's egg have required a much longer time to achieve a comparable effective clinical standard. Only recently, with the development of vitrification of oocytes, the clinical standard was recognized, and since 2013 when the label "experimental" was removed, the freezing of oocytes could be regarded as an established method, and its use extended into clinical practice for fertility preservation and "social freezing" [4].

Many modern women postpone childbearing in order to complete their education, get their career on solid footing or find the right partner with whom they want to share their life. As a result, increasing numbers of women find themselves over age 35 and confronting fertility challenges. Improvements in oocyte cryopreservation offer women the possibility of greater control of their reproductive future by potentially extending their fertility. While women with a family history of premature ovarian failure, repetitive ovarian cysts, living in an area with high exposure to pesticides or heavy metals or undergoing exposure to chemical or biological warfare due to military service may also consider egg freezing, the largest numbers of women considering social egg freezing are likely to be those who either desire or foresee delaying their childbearing years.

A recent paper explored the benefits and moral arguments in favor of women and couples freezing eggs for social reasons (social IVF) [5]. Social IVF promotes equal participation by women in employment; it offers women 
more time to choose a partner; it provides better opportunities for the child as it allows couples more time to become financially stable; it may reduce the risk of genetic and chromosomal abnormality; it allows women and couples to have another child if circumstances change; it offers an option to women and children at risk of ovarian failure; it may increase the egg and embryo pool [5]. These are strong arguments based on equal concern and respect for women, which require that women have access to this new technology.

A small number of studies from the USA and Europe have provided some data on the profile and characteristics of women who have undergone oocyte cryopreservation for what has been termed elective, social or non-medical reasons; however, little is known in a UK context about which women are undergoing oocyte cryopreservation or their reproductive intentions and actions after the procedure. Drawing on data from an exploratory study of 23 UK resident women who had undergone social oocyte cryopreservation, the demographic profile of these women and their reproductive intentions and actions are discussed in a recent British paper [6], as well as their attitudes and intentions toward their cryopreserved oocytes should they never require them in treatment. The study found that, at the time of oocyte cryopreservation, women were on average 36.7 years of age, were university educated, with $65 \%$ of the sample holding further postgraduate or professional qualifications. Fifty-seven percent of the participants were in professional employment. All participants identified as heterosexual, and $87 \%$ were not in a relationship at the time of cryopreserving their oocytes. Most $(88 \%)$ participants stated that they would donate unwanted oocytes to research or to other women for use in fertility treatment should they never require them [6].

Potdar et al.'s [7] systematic review and meta-analysis provide evidence-based information about oocyte survival and fertility outcomes post-warming to help women to make informed choice. All randomized and non-randomized, controlled and prospective cohort studies using oocyte vitrification were included in their review. The primary outcome measure was ongoing pregnancy rate/ warmed oocyte. Sensitivity analyses for donor and nondonor oocyte studies were performed. Proportional metaanalysis of 17 studies, using a random-effects model, showed pooled ongoing pregnancy and clinical pregnancy rates per warmed oocyte of $7 \%$. Oocyte survival, fertilization, cleavage, clinical pregnancy and ongoing pregnancy rates per warmed oocyte were higher in donor versus non-donor studies. Comparing vitrified with fresh oocytes, no statistically significant difference was observed in fertilization, cleavage and clinical pregnancy rates, but ongoing pregnancy rate was reduced in the vitrified group (odds ratio 0.74), with heterogeneity between studies [7].

Wang et al.'s study [8] considered whether it is best to freeze immature human oocytes at the germinal vesicle (GV) stage, prior to in vitro maturation (IVM) or at metaphase-II (M-II), after IVM. Sibling GV-stage oocytes from stimulated ICSI cycles were allocated to freezing either prior to $(n=109)$ or after $(n=107)$ IVM. When frozen as GVs, oocytes exhibited decreased maturation and increased spontaneous activation, suggesting that it is best to freeze oocytes at M-II [8].

A survey was performed in Belgium among 1914 women of reproductive age (21-40 years) to assess public attitudes toward the phenomenon called "social oocyte freezing" [9]. The electronic questionnaire was completed by 1049 women, giving a response rate of $55 \%$, and 25 were excluded as they were incomplete/inconsistent. The results demonstrated that $31.5 \%$ of respondents consider themselves as potential social oocyte freezers, of which $3.1 \%$ would definitely consider the procedure. Just over half of the women $(51.8 \%)$ would not consider the procedure, while $16.7 \%$ indicated they had no opinion. Potential oocyte freezers are characterized by a higher number of desired children and more openness to oocyte donation. The decision to actually embark on such treatment would primarily depend on conditions, such as the procedure not affecting their natural fertility and the health of future children [9].

Tan et al. [10] conducted a cross-sectional survey on 129 female medical students in Singapore to assess their mindset and attitudes toward fertility and social oocyte freezing. An anonymous online survey was conducted among female medical students in Singapore. The desired sample size was 100 participants. Their awareness of the existence of social oocyte freezing was first assessed. An information leaflet was provided subsequently, followed by a more detailed questionnaire. The questions focused on their awareness of age-related fertility decline and their intentions for social oocyte freezing if made available. One hundred and twenty-nine female students participated in the electronic survey, of whom $36.4 \%$ had heard of social oocyte freezing. Of these, $70 \%$ had personally considered taking up this option. However, after reading the information leaflet, only $48.9 \%$ would still consider this option. Of the total, $89.9 \%$ considered themselves too old for pregnancy after the age of 35 years, $37.2 \%$ would delay family planning for their career, $45.7 \%$ would consider social oocyte freezing to postpone family planning for their career, $46.5 \%$ would consider oocyte freezing if they had no suitable partners yet, $50.4 \%$ may consider freezing their eggs after the age of 30 years and $71.3 \%$ may be more amenable to oocyte freezing if government subsidy is available. The authors hypothesized that social oocyte 
freezing may be a viable option for single young women who wish to delay childbearing for "reproductive insurance," so long as this is done with appropriate informed consent with nondirective counseling [10].

A follow-up study in 2014 included a cohort of 85 women who cryopreserved their oocytes between 2009 and 2012 [11]. A total of 68 women, followed up for an average 25.3 months, returned the questionnaire (response rate: $80 \%$ ). None of the women had used her cryopreserved oocytes although 16 women had tried to conceive. Of these women, eight were trying to conceive naturally, five had conceived naturally within 2 months and three had conceived with assisted reproduction not requiring cryopreserved oocytes (two women with conventional IVF because of tubal pathology and endometriosis and one woman with IUI because of polycystic ovary syndrome). Three out of the eight pregnancies had resulted in live births, two resulted in miscarriages and three were ongoing. Most women (71\%) intended to conceive with their cryopreserved oocytes as a last resource option [11]. After a mean follow-up of 2 years, none of the women with a medical reason to cryopreserved oocytes had used her oocytes. Women who were trying to conceive during follow-up were doing so without using their stored oocytes. It is unclear whether starting assisted reproduction while having cryopreserved oocytes is the most appropriate clinical decision. These findings emphasize the relevance of taking the chances of natural conception into account in counseling women about cryopreservation of oocytes.

Recently, the Israel National Bioethics Council (INBC) issued recommendations permitting egg freezing to prevent both disease- and age-related fertility declines [12]. The INBC report forms the basis of Israel's new policy, being one of the first countries to regulate and authorize egg freezing for what it considers to be non-medical (i.e., social) uses. The ethical discussion in the INBC report is reviewed and compared with the scant ethical discourse in the academic literature on egg freezing as a means of preventing age-related loss of fertility. Shkedi-Rafid and Hashiloni-Dolev [12] argued that both the INBC recommendations and the bioethical academic discourse on egg freezing are grounded in liberal ideology, which views technology as primarily enabling. Accordingly, they promote "individual autonomy" as exercised through informed consent.

Finally, a very recent literature review from Zhang et al. [13] summarized that vitrification of mature oocytes obtained better clinical outcomes and did not increase the risks of DNA damage, spindle configuration, embryonic aneuploidy and genomic imprinting as compared with fresh and slow-freezing procedures, respectively.

\section{Recent Advances}

Egg cryopreservation by vitrification has become one of the most important assisted human reproduction technologies. Although reports indicate that development and implantation of human embryos derived from frozen donor eggs are comparative to fresh eggs, it is still unknown whether egg vitrification increases chromosomal abnormalities in eggs, which in turn causes formation of embryonic aneuploidy. Therefore, in a recent study, Deng and Wang [14] evaluated the aneuploidy formation in the blastocysts derived from frozen donor eggs and also the efficiency of egg vitrification as an advanced technology for egg cryopreservation. In this study, donated human eggs from young women were cryopreserved by vitrification, and PGS was performed in the resulted blastocysts by DNA microarray. A total of 764 frozen eggs from 75 egg thawing cycles were warmed, and 38 blastocysts were biopsied for PGS before embryo transfer. A $97.1 \%$ of egg survival rate was obtained, and $59.1 \%$ of embryos developed to blastocyst stage. After biopsy and PGS, it was found that $84.2 \%$ of blastocysts were euploid and $15.8 \%$ were aneuploid. Aneuploidy rates varied among donors. Transfers of blastocysts without PGS resulted in higher clinical pregnancy and implantation rates as compared with transfer of blastocysts with PGS [14]. Clinical pregnancy rate was not improved by PGS of embryos resulting from donor eggs, indicating that PGS may not be necessary for embryos derived from donor eggs in most cases.

\section{Conclusions}

The chance of a live birth is similar for vitrified and "fresh" eggs used in IVF treatment. The two most important factors that determine the chance of having a baby from frozen eggs are the woman's age when the eggs were frozen and the number of eggs that are stored. The number and quality of the eggs that develop when the ovaries are stimulated decline with increasing age. A woman in her early thirties might have 15-20 eggs available for freezing after the hormone stimulation, but for women in their late thirties and early forties the number is usually much lower. Also, as women age they are more likely to have eggs with chromosomal abnormalities. The number of eggs available for freezing and their quality is important because in every step there is a risk that some are lost. Of the eggs that are retrieved, some may not be suitable for freezing, some may not survive the freezing and thawing processes and some may not fertilize or develop into normal embryos. Of the embryos that are transferred, only some will result in a pregnancy, and some 
pregnancies miscarry. Current literature suggests that vitrified oocytes produce superior IVF results to slow-frozen oocytes and may yield comparable outcomes to IVF with fresh oocytes in certain patient populations. Patients at risk of infertility due to disease- or age-related decline or oocyte donation programs, couples who fail to produce semen when required for IVF and patients with legal or ethical reasons against embryo cryopreservation may access cryopreserved oocytes. Freezing eggs also avoids some of the moral objections associated with freezing embryos.

\section{References}

1. Chen C. Pregnancy after human oocyte cryopreservation. Lancet. 1986;1(8486):884-6.

2. American Cancer Society. Cancer facts and figures 2015. Atlanta: American Cancer Society. http://www.cancer.org/ research/cancerfactsstatistics/allcancerfactsfigures/index. Retrieved on 25 Sept 2015.

3. Tsafrir A, Haimov-Kochman R, Margalioth EJ, et al. Ovarian stimulation for oocyte cryopreservation for prevention of agerelated fertility loss: one in five is a low responder. Gynecol Endocrinol. 2015;18:1-4.

4. Rodriguez-Wallberg KA. Clinical aspects and perinatal outcomes after cryopreservation of embryos and gametes. Minerva Ginecol. 2015;67(2):207-15.

5. Goold I, Savulescu J. In favour of freezing eggs for non-medical reasons. Bioethics. 2009;23(1):47-58. doi:10.1111/j.1467-8519. 2008.00679.x.
6. Baldwin K, Culley L, Hudson N, et al. Oocyte cryopreservation for social reasons: demographic profile and disposal intentions of UK users. Reprod Biomed Online. 2015;31(2):239-45. doi: 10.1016/j.rbmo.2015.04.010.

7. Potdar N, Gelbaya TA, Nardo LG. Oocyte vitrification in the 21st century and post-warming fertility outcomes: a systematic review and meta-analysis. Reprod Biomed Online. 2014;29(2):159-76. doi:10.1016/j.rbmo.2014.03.024.

8. Wang H, Racowsky C, Combelles CM. Is it best to cryopreserve human cumulus-free immature oocytes before or after in vitro maturation? Cryobiology. 2012;65(2):79-87. doi:10.1016/j.cryobiol. 2012.06.001.

9. Stoop D, Nekkebroeck J, Devroey P. A survey on the intentions and attitudes towards oocyte cryopreservation for non-medical reasons among women of reproductive age. Hum Reprod. 2011;26(3):655-61. doi:10.1093/humrep/deq367.

10. Tan SQ, Tan AW, Lau MS, et al. Social oocyte freezing: a survey among Singaporean female medical students. J Obstet Gynaecol Res. 2014;40(5):1345-52. doi:10.1111/jog. 12347.

11. Dahhan T, Dancet EA, Miedema DV, et al. Reproductive choices and outcomes after freezing oocytes for medical reasons: a follow-up study. Hum Reprod. 2014;29(9):1925-30. doi:10.1093/ humrep/deu137.

12. Shkedi-Rafid S, Hashiloni-Dolev Y. Egg freezing for non-medical uses: the lack of a relational approach to autonomy in the new Israeli policy and in academic discussion. $\mathrm{J}$ Med Ethics. 2012;38(3):154-7. doi:10.1136/medethics-2011-100088.

13. Zhang L, Yan LY, Zhi X, et al. Female fertility: is it safe to “freeze?". Chin Med J (Engl). 2015;128(3):390-7. doi:10.4103/ 0366-6999.150115.

14. Deng A, Wang WH. Assessment of aneuploidy formation in human blastocysts resulting from cryopreserved donor eggs. Mol Cytogenet. 2015;8:12. doi:10.1186/s13039-015-0117-8. 